\title{
A tomography of the GREM: beyond the REM conjecture ${ }^{1}$
}

\author{
Anton Bovier ${ }^{2}$ \\ Weierstraß-Institut \\ für Angewandte Analysis und Stochastik \\ Mohrenstrasse 39, 10117 Berlin, Germany \\ and \\ Institut für Mathematik \\ Technische Universität Berlin \\ Strasse des 17. Juni 136, 12623 Berlin, Germany \\ Irina Kurkova ${ }^{3}$ \\ Laboratoire de Probabilités et Modèles Aléatoires \\ Université Paris 6 \\ 4, place Jussieu, B.C. 188 \\ 75252 Paris, Cedex 5, France
}

\begin{abstract}
Recently, Bauke and Mertens conjectured that the local statistics of energies in random spin systems with discrete spin space should in most circumstances be the same as in the random energy model. This was proven in a large class of models for energies that do not grow too fast with the system size. Considering the example of the generalized random energy model, we show that the conjecture breaks down for energies proportional to the volume of the system, and describe the far more complex behavior that then sets in.

Keywords: level statistics, random energy model, generalized random energy model, extreme value theory, disordered systems, spin glasses

AMS Subject Classification: 60G70, 82B45
\end{abstract}

\footnotetext{
${ }^{1}$ Research supported in part by the DFG in the Dutch-German Bilateral Research Group "Mathematics of Random Spatial Models from Physics and Biology" and by the European Science Foundation in the Programme RDSES.

2e-mail: bovier@wias-berlin.de

${ }^{3}$ e-mail: kourkova@ccr.jussieu.fr 


\section{Introduction.}

In a recent paper [BaMe], Bauke and Mertens have formulated an interesting conjecture on the behavior of local energy level statistics in disordered systems. Roughly speaking, their conjecture can be formulated as follows. Consider a random Hamiltonian, $H_{N}(\sigma)$, i.e. a random function from some product space, $\mathcal{S}^{N}$, where $\mathcal{S}$ is a finite space, typically $\{-1,1\}$, to the real numbers. We may assume for simplicity that $\mathbb{E} H_{N}(\sigma)=0$. In such a situation, for typical $\sigma, H_{N}(\sigma) \sim \sqrt{N}$, while $\sup _{\sigma} H_{N}(\sigma) \sim N$. Bauke and Mertens then ask the following question: Given a fixed number, $E$, what are the statistics of the values $N^{-1 / 2} H_{N}(\sigma)$ that are closest to this number $E$, and how are configurations, $\sigma$, for which these good approximants of $E$ are realized, distributed on $\mathcal{S}^{N}$ ? Their conjectured answer, which at first glance seems rather surprising, is simple: find $\delta_{N, E}$ such that $\mathbb{P}\left(\left|N^{-1 / 2} H_{N}(\sigma)-E\right| \leq b \delta_{N, E}\right) \sim|\mathcal{S}|^{-N_{b}}$ for any constant $b>0$; then the collection of points $\delta_{N, E}^{-1}\left|N^{-1 / 2} H_{N}(\sigma)-E\right|$ over all $\sigma \in \mathcal{S}^{N}$ converges to a Poisson point process on $\mathbb{R}_{+}$, with intensity measure the Lebesgue measure. Furthermore, for any finite $k$, the $k$-tuple of configurations $\sigma^{1}, \sigma^{2}, \ldots, \sigma^{k}$, where the $k$ best approximations are realized, is such that all of its elements have maximal Hamming distance between each other. In other words, the asymptotic behavior of these best approximants of $E$ is the same, as if the random variables $H_{N}(\sigma)$ were all independent Gaussian random variables with zero mean and variance $N$, i.e. as if we were dealing with the random energy model (REM) [Der1]; for this reason, Bauke and Mertens call this phenomenon "universal REM like behavior".

This conjecture was proven recently [BK2] in a wide class of models, including mean field models and short range spin glass models. In the case of Gaussian interactions, it was shown to hold even for energies that diverge with the volume of the system, $N$, as $E_{N}=c N^{\alpha}$, for $0 \leq \alpha<\alpha_{0}$, where $\alpha_{0}$ is model dependent.

Is is rather clear that the conjecture must break down in general for $\alpha$ such that $c N^{\alpha}$ is of the order of the maximum of $H_{N}(\sigma)$. It is a natural question to ask what will happen in this

regime. Naturally, the answers will become model dependent, and in general very difficult to obtain. The only (non-trivial) models where we are able to carry out such an analysis in detail are the so-called generalized random energy models (GREMs) of Derrida [Der2]. In these models, the extremal process was analyzed in full in [BK1]. The result we obtain gives a somewhat extreme microcanonical picture of the GREM, exhibiting in a somewhat tomographic way the distribution of states in a tiny vicinity of any value of the energy.

Let us briefly recall the definition of the GREM. We consider parameters $\alpha_{0}=1<$ 
$\alpha_{1}, \ldots, \alpha_{n}<2$ with $\prod_{i=1}^{n} \alpha_{i}=2, a_{0}=0<a_{1}, \ldots, a_{n}<1, \sum_{i=1}^{n} a_{i}=1$. Let $\Sigma_{N}=\{-1,1\}^{N}$ be the space of $2^{N}$ spin configurations $\sigma$. Let $X_{\sigma_{1} \cdots \sigma_{l}}, l=1, \ldots, n$, be independent standard Gaussian random variables indexed by configurations $\sigma_{1} \ldots \sigma_{l} \in\{-1,1\}^{N \ln \left(\alpha_{1} \cdots \alpha_{l}\right) / \ln 2}$. We define the Hamiltonian of the GREM as $H_{N}(\sigma) \equiv \sqrt{N} X_{\sigma}$, with

$$
X_{\sigma} \equiv \sqrt{a_{1}} X_{\sigma_{1}}+\cdots+\sqrt{a_{n}} X_{\sigma_{1} \cdots \sigma_{n}} .
$$

Then $\operatorname{cov}\left(X_{\sigma}, X_{\sigma^{\prime}}\right)=A\left(d_{N}\left(\sigma, \sigma^{\prime}\right)\right)$, where $d_{N}\left(\sigma, \sigma^{\prime}\right)=N^{-1}\left[\min \left\{i: \sigma_{i} \neq \sigma_{i}^{\prime}\right\}-1\right]$, and $A(x)$ is a right-continuous step function on $[0,1]$, such that, for any $i=0,1, \ldots, n, A(x)=a_{0}+\cdots+a_{i}$, for $x \in\left[\ln \left(\alpha_{0} \alpha_{1}, \cdots \alpha_{i}\right) / \ln 2, \ln \left(\alpha_{0} \alpha_{1}, \cdots \alpha_{i+1}\right) / \ln 2\right)$.

Set $J_{0} \equiv 0$, and, define, for $l>0$,

$$
J_{l}=\min \left\{n \geq J>J_{l-1}: \frac{\ln \left(\alpha_{J_{l-1}+1} \cdots \alpha_{J}\right)}{a_{J_{l-1}+1}+\cdots+a_{J}}<\frac{\ln \left(\alpha_{J+1} \cdots \alpha_{m}\right)}{a_{J+1}+\cdots+a_{m}} \forall m \geq J+1\right\} .
$$

up to $J_{k}=n$. Then, the $k$ segments connecting the points $\left(a_{0}+\cdots+a_{J_{l}}, \ln \left(\alpha_{0} \alpha_{1} \cdots \alpha_{J_{l}}\right) / \ln 2\right)$, for $l=0,1, \ldots, k$ form the concave hull of the graph of the function $A(x)$. Let

$$
\bar{a}_{l}=a_{J_{l-1}+1}+a_{J_{l-1}+2}+\cdots+a_{J_{l}}, \quad \bar{\alpha}_{l}=\alpha_{J_{l-1}+1} \alpha_{J_{l-1}+2} \cdots \alpha_{J_{l}} .
$$

Then

$$
\frac{\ln \bar{\alpha}_{1}}{\bar{a}_{1}}<\frac{\ln \bar{\alpha}_{2}}{\bar{a}_{2}}<\cdots<\frac{\ln \bar{\alpha}_{k}}{\bar{a}_{k}} .
$$

Moreover, as it is shown in Proposition 1.4 of [BK1], for any $l=1, \ldots, k$, and for any $J_{l-1}+1 \leq i<J_{l}$, we have $\ln \left(\alpha_{J_{l-1}+1} \cdots \alpha_{i}\right) /\left(a_{J_{l-1}+1}+\cdots+a_{i}\right) \geq \ln \left(\bar{\alpha}_{l}\right) / \bar{a}_{l}$. Hence

$$
\frac{\ln \bar{\alpha}_{l}}{\bar{a}_{l}}=\min _{j=J_{l-1}+1, J_{l-1}+2, \ldots, n} \frac{\ln \left(\alpha_{J_{l-1}+1} \ldots \alpha_{j}\right)}{a_{J_{l-1}+1}+\cdots+a_{j}} .
$$

To formulate our results, we also need to recall from [BK1] (Lemma 1.2) the point process of Poisson cascades $\mathcal{P}^{l}$ on $\mathbb{R}^{l}$. It is best understood in terms of the following iterative construction. If $l=1, \mathcal{P}^{1}$ is the Poisson point process on $\mathbb{R}^{1}$ with the intensity measure $K_{1} e^{-x} d x$. To construct $\mathcal{P}^{l}$, we place the process $\mathcal{P}^{l-1}$ on the plane of the first $l-1$ coordinates and through each of its points draw a straight line orthogonal to this plane. Then we put on each of these lines independently a Poisson point process with intensity measure $K_{l} e^{-x} d x$. These points on $\mathbb{R}^{l}$ form the process $\mathcal{P}^{l}$. The constants $K_{1}, \ldots, K_{l}>0$ (that are different from 1 only in some degenerate cases) are defined in the formula (1.14) of [BK1]. 
We will also need the following facts concerning $\mathcal{P}^{l}$ from Theorem 1.5 of [BK1]. Let $\gamma_{1}>\gamma_{2}>\cdots>\gamma_{l}>0$. There exists a constant $h>0$, such that, for all $y>0$,

$\mathbb{P}\left(\exists\left(x_{1}, \ldots, x_{l}\right) \in \mathcal{P}^{l}, \exists j=1, \ldots, l: \gamma_{1} x_{1}+\gamma_{2} x_{2}+\cdots+\gamma_{j} x_{j}>\left(\gamma_{1}+\cdots+\gamma_{j}\right) y\right) \leq \exp (-h y)$

Here and below we identify the measure $\mathcal{P}^{l}$ with its support, when suitable. Furthermore, for any $y \in \mathbb{R}$,

$$
\#\left\{\left(x_{1}, \ldots, x_{l}\right) \in \mathcal{P}^{l}: x_{1} \gamma_{1}+\cdots+x_{l} \gamma_{l}>y\right\}<\infty \text { a.s. }
$$

Moreover, let $\beta>0$ be such that $\beta \gamma_{1}>\cdots>\beta \gamma_{l}>1$. The integral

$$
\Lambda_{l}=\int_{\mathbb{R}^{l}} e^{\beta\left(\gamma_{1} x_{1}+\cdots \gamma_{l} x_{l}\right)} \mathcal{P}^{l}\left(d x_{1}, \ldots, d x_{l}\right) .
$$

is understood as $\lim _{y \rightarrow-\infty} I_{l}(y)$ with

$$
\begin{aligned}
& I_{l}(y)=\int_{\substack{\left(x_{1}, \ldots, x_{l}\right) \in \mathbb{R}^{l}: \\
\exists i, 1 \leq i \leq l: \gamma_{1} x_{1}+\cdots+\gamma_{i} x_{i}>\left(\gamma_{1}+\cdots+\gamma_{i}\right) y}} e^{\beta\left(\gamma_{1} x_{1}+\cdots+\gamma_{l} x_{l}\right)} \mathcal{P}^{l}\left(d x_{1}, \ldots, d x_{l}\right) \\
& =\sum_{j=1}^{l} \int_{\substack{\left.\left(x_{1}, \ldots, x_{l}\right) \in \mathbb{R} l: \\
\forall i=1, \ldots, j-1: \gamma_{1} x_{1}+\cdots+\gamma_{i} \leq<\gamma_{1}+\cdots+\gamma_{i}\right) y \\
\gamma_{1} x_{1}+\cdots+\gamma_{j} x_{j}>\left(\gamma_{1}+\cdots+\gamma_{j}\right) y}} e^{\beta\left(\gamma_{1} x_{1}+\cdots+\gamma_{l} x_{l}\right)} \mathcal{P}^{l}\left(d x_{1}, \ldots, d x_{l},\right) .
\end{aligned}
$$

It is finite, a.s., by Proposition 1.8 of [BK1]. To keep the paper self-contained, let us recall how this fact can be established by induction starting from $l=1$. The integral (1.8), in the case $l=1$, is understood as $\lim _{y \rightarrow-\infty} I_{1}(y)$. Here $I_{1}(y)=\int_{y}^{\infty} e^{\beta \gamma_{1} x_{1}} \mathcal{P}_{1}(d x)$ is finite, a.s., since $\mathcal{P}_{1}$ contains a finite number of points on $[y, \infty[$, a.s. Furthermore, by [BKL] or Proposition 1.8 of [BK1], $\lim _{y \rightarrow-\infty} I_{1}(y)$ is finite, a.s., since $\mathbb{E} \sup _{y^{\prime} \leq y}\left(I_{1}\left(y^{\prime}\right)-I_{1}(y)\right)$ converges to zero exponentially fast, as $y \rightarrow-\infty$, provided that $\beta \gamma_{1}>1$. If $l \geq 1$, each term in the representation (1.9) is determined and finite, a.s., by induction. In fact, to see this for the $j$ th term, given any realization of $\mathcal{P}^{l}$ in $\mathbb{R}^{l}$, take its projection on the plane of the first $j$ coordinates. Then by (1.7), there exists only a finite number of points $\left(x_{1}, \ldots, x_{j}\right)$ of $\mathcal{P}^{j}$, such that $\gamma_{1} x_{1}+\cdots+\gamma_{j} x_{j}>\left(\gamma_{1}+\cdots+\gamma_{j}\right) y$, a.s. Whenever the first $j$ coordinates of a point of $\mathcal{P}^{l}$ in $\mathbb{R}^{l}$ are fixed, the remaining $l-j$ coordinates are distributed as $\mathcal{P}^{l-j}$ on $\mathbb{R}^{l-j}$. Then the integral over the function $e^{\beta\left(\gamma_{j+1} x_{j+1}+\cdots+\gamma_{l} x_{l}\right)}$ over these coordinates is defined by induction and is finite, a.s., provided that $\beta \gamma_{j+1}>\cdots>\beta \gamma_{l}>1$. Thus the $j$ th term in (1.9) is the sum of an a.s. finite number of terms and each of them is a.s. finite. Finally, again by 
Proposition 1.8 of $[\mathrm{BK} 1], \lim _{y \rightarrow-\infty} I_{l}(y)$ is finite, a.s., since $\mathbb{E} \sup _{y^{\prime} \leq y}\left(I_{l}\left(y^{\prime}\right)-I_{l}(y)\right) \rightarrow 0$ as $y \rightarrow-\infty$ exponentially fast provided that $\beta \gamma_{1}>\cdots>\beta \gamma_{l}>1$.

Let us define the constants $d_{l}, l=0,1, \ldots, k$, where $d_{0}=0$ and

$$
d_{l} \equiv \sum_{i=1}^{l} \sqrt{\bar{a}_{i} 2 \ln \bar{\alpha}_{i}}
$$

Finally, we define the domains $\mathcal{D}_{l}$, for $l=0, \ldots, k-1$, as

$$
\mathcal{D}_{l} \equiv\left\{|y|<d_{l}+\sqrt{\frac{2 \ln \bar{\alpha}_{l+1}}{\bar{a}_{l+1}}} \sum_{j=l+1}^{k} \bar{a}_{j}\right\} \text {. }
$$

It is not difficult to verify that $\mathcal{D}_{0} \subset \mathcal{D}_{1} \subset \cdots \subset \mathcal{D}_{k-1}$. We are now ready to formulate the main result of this paper.

Theorem 1.1: Let a sequence $c_{N} \in \mathbb{R}$ be such that $\lim \sup _{N \rightarrow \infty} c_{N} \in \mathcal{D}_{0}$ and $\lim \inf _{N \rightarrow \infty} c_{N} \in$ $\mathcal{D}_{0}$. Then, the point process

$$
\mathcal{M}_{N}^{0}=\sum_{\sigma \in \Sigma_{N}} \delta_{\left\{2^{N+1}(2 \pi)^{-1 / 2} e^{-c_{N}^{2} N / 2}\left|X_{\sigma}-c_{N} \sqrt{N}\right|\right\}}
$$

converges to the Poisson point process with intensity measure the Lebesgue measure.

Let, for $l=1, \ldots, k-1, c \in \mathcal{D}_{l} \backslash \overline{\mathcal{D}_{l-1}}$ (where $\overline{\mathcal{D}_{l-1}}$ is the closure of $\mathcal{D}_{l-1}$ ). Define

$$
\begin{gathered}
\tilde{c}_{l}=|c|-d_{l}, \\
\beta_{l}=\frac{\tilde{c}_{l}}{\bar{a}_{l+1}+\cdots+\bar{a}_{k}}, \quad \gamma_{i}=\sqrt{\bar{a}_{i} /\left(2 \ln \bar{\alpha}_{i}\right)}, \quad i=1, \ldots, l,
\end{gathered}
$$

and

$$
R_{l}(N)=\frac{2\left(\bar{\alpha}_{l+1} \cdots \bar{\alpha}_{k}\right)^{N} \exp \left(-N \tilde{c}_{l} \beta_{l} / 2\right)}{\sqrt{2 \pi\left(\bar{a}_{l+1}+\cdots+\bar{a}_{k}\right)}} \prod_{j=1}^{l}\left(4 N \pi \ln \bar{\alpha}_{j}\right)^{-\beta_{l} \gamma_{j} / 2} .
$$

Then, the point process

$$
\mathcal{M}_{N}^{l}=\sum_{\sigma \in \Sigma_{N}} \delta_{\left\{R_{l}(N)\left|\sqrt{a_{1}} X_{\sigma_{1}}+\cdots+\sqrt{a_{n}} X_{\sigma_{1} \ldots \sigma_{n}}-c \sqrt{N}\right|\right\}}
$$

converges to mixed Poisson point process on $[0, \infty[:$ given a realization of the random variable $\Lambda_{l}$, its intensity measure is $\Lambda_{l} d x$. The random variables $\Lambda_{l}$ is defined in terms of the Poisson cascades $\mathcal{P}_{l}$ via

$$
\Lambda_{l}=\int_{\mathbb{R}^{l}} e^{\beta_{l}\left(\gamma_{1} x_{1}+\cdots \gamma_{l} x_{l}\right)} \mathcal{P}^{l}\left(d x_{1}, \ldots, d x_{l}\right)
$$


The next section will be devoted to the proof of this result. Before doing this, we conclude the present section with a heuristic interpretation of the main result.

Let us first look at (1.12). This statement corresponds to the REM-conjecture of Bauke and Mertens [BaMe]. It is quite remarkable that this conjecture holds in the case of the GREM for energies of the form $c N$ (namely for $c \in \mathcal{D}_{0}$ ).

In the REM [Der1], $X_{\sigma}$ are $2^{N}$ independent standard Gaussian random variables and a statement (1.12) would hold for all $c$ with $|c|<\sqrt{2 \ln 2}$ : it is a well known result from the theory of independent random variables [LLR]. The value $c=\sqrt{2 \ln 2}$ corresponds to the maximum of $2^{N}$ independent standard Gaussian random variables, i.e., $\max _{\sigma \in \Sigma_{N}} N^{-1 / 2} X_{\sigma} \rightarrow$ $\sqrt{2 \ln 2}$ a.s. Therefore, at the level $c=\sqrt{2 \ln 2}$, one has the emergence of the extremal process. More precisely, the point process

$$
\sum_{\sigma \in \Sigma_{N}} \delta_{\left\{\sqrt{2 N \ln 2}\left(X_{\sigma}-\sqrt{2 N \ln 2}+\ln (4 \pi N \ln 2) / \sqrt{8 N \ln 2}\right)\right\}}
$$

that is commonly written as $\sum_{\sigma \in \Sigma_{N}} \delta_{u_{N}^{-1}\left(X_{\sigma}\right)}$ with

$$
u_{N}(x)=\sqrt{2 N \ln 2}-\frac{\ln (4 \pi N \ln 2)}{2 \sqrt{2 N \ln 2}}+\frac{x}{\sqrt{2 N \ln 2}}
$$

converges to the Poisson point process $\mathcal{P}^{1}$ defined above (see e.g. [LLR]). For $c>\sqrt{2 \ln 2}$, the probability that one of the $X_{\sigma}$ will be outside of the domain $\{|x|<c \sqrt{N}\}$, goes to zero, and thus it makes no sense to consider such levels.

In the GREM, $N^{-1 / 2} \max _{\sigma \in \Sigma_{N}} X_{\sigma}$ converges to the value $d_{k} \in \partial D_{k-1}$ (1.10) (see Theorem 1.5 of [BK1]) that is generally smaller than $\sqrt{2 \ln 2}$. Thus it makes no sense to consider levels with $c \notin \bar{D}_{k-1}$. However, the REM-conjecture is not true for all levels in $\mathcal{D}_{k-1}$, but only in the smaller domain $\mathcal{D}_{0}$.

To understand the statement of the theorem outside $\mathcal{D}_{0}$, we need to recall how the extremal process in the GREM is related to the Poisson cascades introduced above. Let us set $\Sigma_{N w_{l}} \equiv$ $\{-1,1\}^{N w_{l}}$ where

$$
w_{l}=\ln \left(\bar{\alpha}_{1} \cdots \bar{\alpha}_{l}\right) / \ln 2
$$

with the notation (1.3). Let us also define the functions

$$
U_{l, N}(x) \equiv N^{1 / 2} d_{l}-N^{-1 / 2} \sum_{i=1}^{l} \gamma_{i} \ln \left(4 \pi N \ln \bar{\alpha}_{i}\right) / 2+N^{-1 / 2} x
$$


with the notations (1.3), (1.10), (1.14), and set

$$
\widehat{X}_{\sigma}^{j} \equiv \sum_{i=1}^{j} \sqrt{a}_{i} X_{\sigma_{1} \ldots \sigma_{i}}, \quad \check{X}_{\sigma}^{j} \equiv \sum_{i=j+1}^{n} \sqrt{a}_{i} X_{\sigma_{1} \ldots \sigma_{i}} .
$$

From what was shown in $[\mathrm{BK} 1]$, for any $l=1, \ldots, k$, the point process,

$$
\mathcal{E}_{l, N} \equiv \sum_{\hat{\sigma} \in \Sigma_{N} w_{l}} \delta_{U_{l, N}^{-1}\left(\widehat{X}_{\hat{\sigma}}^{J_{l}}\right)}
$$

converges in law to the Poisson cluster process, $\mathcal{E}_{l}$, given in terms of the Poisson cascade, $\mathcal{P}^{l}$, as

$$
\mathcal{E}_{l} \equiv \int_{\mathbb{R}^{l}} \mathcal{P}^{(l)}\left(d x_{1}, \ldots, d x_{l}\right) \delta_{\sum_{i=1}^{l} \gamma_{i} x_{i}} .
$$

In view of this observation, we can re-write the definition of the process $\mathcal{M}_{N}^{l}$ as follows:

$$
\mathcal{M}_{N}^{l}=\sum_{\hat{\sigma} \in \Sigma_{w_{l} N}} \sum_{\check{\sigma} \in \Sigma_{\left(1-w_{l}\right) N}} \delta_{\left\{R_{l}(N)\left|\check{X}_{\tilde{\sigma} \check{\sigma}}^{J_{l}}-\sqrt{N}\left[|c|-d_{l}-N^{-1}\left(\Gamma_{l, N}-U_{l, N}^{-1}\left(\widehat{X}_{\tilde{\sigma}}^{J_{l}}\right)\right)\right]\right|\right\}}
$$

with the abbreviation

$$
\Gamma_{l, N} \equiv \sum_{i=1}^{l} \gamma_{i} \ln \left(4 \pi N \ln \bar{\alpha}_{i}\right) / 2
$$

( $c$ is replaced by $|c|$ due to the symmetry of the standard Gaussian distribution). The normalizing constant, $R_{l}(N)$, is chosen such that, for any finite value, $U$, the point process

$$
\sum_{\check{\sigma} \in \Sigma_{\left(1-w_{l}\right) N}} \delta_{\left\{R_{l}(N)\left|\check{X}_{\tilde{\sigma} \check{\sigma}}^{J_{l}}-\sqrt{N}\left[|c|-d_{l}-N^{-1}\left(\Gamma_{l, N}-U\right)\right]\right|\right\}}
$$

converges to the Poisson point processes on $\mathbb{R}_{+}$, with intensity measure given by $e^{U}$ times Lebesgue measure, which is possible precisely because $c \in \mathcal{D}_{l} \backslash \overline{\mathcal{D}_{l-1}}$, that is $|c|-d_{l}$ is smaller that the a.s. limit of $N^{-1 / 2} \max _{\check{\sigma} \in \Sigma_{\left(1-w_{l}\right) N}} \check{X}_{\tilde{\sigma} \check{\sigma}}^{J_{l}}$. This is completely analogous to the analysis in the domain $\mathcal{D}_{0}$. Thus each term in the sum over $\hat{\sigma}$ in (1.25) that gives rise to a "finite" $U_{l, N}^{-1}\left(\widehat{X}_{\hat{\sigma}}^{l}\right)$, i.e., to an element of the extremal process of $\widehat{X}_{\hat{\sigma}}^{l}$, gives rise to one Poisson process with a random intensity measure in the limit of $\mathcal{M}_{N}^{l}$. This explains how the statement of the theorem can be understood, and also shows what the geometry of the configurations realizing these mixed Poisson point processes will be.

Let us add that, if $c \in \partial \mathcal{D}_{k-1}$, i.e. $|c|=d_{k}$, then one has the emergence of the extremal point process (1.23) with $l=k$, i.e. $\sum_{\sigma \in \Sigma_{N}} \delta_{\left\{\sqrt{N}\left(X_{\sigma}-d_{k} \sqrt{N}+N^{-1 / 2} \Gamma_{k, N}\right)\right\}}$ converges to (1.24) with $l=k$, see [BK1]. 


\section{Proof of Theorem 1.1.}

Note that (1.17) is finite a.s. since $\gamma_{1}>\cdots>\gamma_{l}$ by (1.4) and $\beta_{l} \gamma_{l}>1$ by the definition of $\beta_{l}$. Note also that $c$ can be replaced by $|c|$ in (1.12) and (1.16) due to the symmetry of the standard Gaussian distribution.

Let $\mathcal{M}_{N}^{l}(A)$ be the number of points of $\mathcal{M}_{N}^{l}$ in a Borel subset $A \subset \mathbb{R}_{+}$. We will show that for any finite disjoint union of intervals, $A=\cup_{q=1}^{p}\left[a_{q}, b_{q}\right)$, the avoidance function converges

$$
\mathbb{P}\left(\mathcal{M}_{N}^{l}(A)=0\right) \rightarrow \mathbb{E} \exp \left(-|A| \Lambda_{l}\right),
$$

where of course $\Lambda_{0}=1$ in the case $l=0$. Note that in that case, the right-hand side is the avoidance function of a Poisson point process with intensity 1, while in all other cases, this is the avoidance function of a mixed Poisson point process.

To conclude the proof in the case $l=0$, it is enough to show that for any segment $A=[a, b)$

$$
\mathbb{E} \mathcal{M}_{N}^{0}(A) \rightarrow(b-a), \quad N \rightarrow \infty
$$

Then the result (1.12) would follow from Kallenberg's theorem, see [Ka] or [LLR].

In the cases $l=1, \ldots, k-1$ we will prove that the family $\left\{\mathcal{M}_{N}^{l}\right\}_{N=1}^{\infty}$ is uniformly tight: by Proposition $9.1 \mathrm{~V}$ of $[\mathrm{DV}]$, this is equivalent to the fact that, for any compact segment, $A=[a, b]$, and for any given $\epsilon>0$, one can find a large enough integer, $R$, such that

$$
\mathcal{P}\left(\mathcal{M}_{N}^{l}(A)>R\right)<\epsilon, \quad \forall N \geq 1 .
$$

Finally, we will show that the limit of any weakly convergent subsequence of $\mathcal{M}_{N}^{l}$ is a simple point process, that is without double points (see Definition 7.1IV in [DV]). Theorem 7.3II of [DV] asserts that a simple point process is uniquely characterized by its avoidance function, which then implies the result (1.16) claimed in Theorem 1.1.

To prove (2.1), we need the following lemma.

Lemma 2.1: Let $A=\cup_{q=1}^{p}\left[a_{q}, b_{q}\right), 0 \leq a_{1}<b_{1}<a_{2}<b_{2}<\cdots<a_{q}<b_{q}$, with $|A|=\sum_{q=1}^{p}\left(b_{q}-a_{q}\right)$. Let $0<f<1, K(N)>0$ be a polynomial in $N$. We write $K(N) f^{N} A \equiv$ $\cup_{q=1}^{p}\left[K(N) f^{N} a_{q}, K(N) f^{N} b_{q}\right)$.

For any $i=1,2, \ldots, n$, any $\epsilon>0, \delta>0$ small enough, and $M>0$, there exists $N_{0}$, such that, for all $N \geq N_{0}$ and for all $y$, such that

$$
\begin{gathered}
\max \left(\max _{m=i+1, \ldots, n} \frac{\left(a_{i}+\cdots+a_{n}\right)\left(2 \ln \alpha_{m}+\cdots+2 \ln \alpha_{n}+2 \ln f+\epsilon\right)}{a_{m}+\cdots+a_{n}},\right. \\
\left.\left(2 \ln \alpha_{i+1}+\cdots+2 \ln \alpha_{n}+2 \ln f+\epsilon\right)\right) \leq y^{2} \leq M
\end{gathered}
$$


the probability,

$$
\mathbb{P}\left(\forall \check{\sigma} \in\{-1,1\}^{N\left(\ln \left(\alpha_{i} \cdots \alpha_{n}\right) / \ln 2\right)}:\left|\frac{\check{X}_{\check{\sigma}}^{i-1}}{\sqrt{a_{i}+\cdots+a_{n}}}-y \sqrt{N}\right| \notin K(N) f^{N} A\right),
$$

with $\check{X}_{\check{\sigma}}^{i-1}$ defined by (1.22), is bounded from above and below, respectively, by

$$
\exp \left(-(1 \pm \delta)|A|(2 \pi)^{-1 / 2} 2 K(N) f^{N} \alpha_{i}^{N} \alpha_{i+1}^{N} \cdots \alpha_{n}^{N} e^{-y^{2} N / 2}\right)
$$

Proof. Let us define the quantity

$P_{N}(i, y, f, K(N)) \equiv \mathbb{P}\left(\exists \check{\sigma} \in\{-1,1\}^{\left(\ln \alpha_{i+1}+\cdots \alpha_{n}\right) / \ln 2}:\left|\frac{\check{X}_{\check{\sigma}}^{i}}{\sqrt{a_{i}+\cdots+a_{n}}}-y \sqrt{N}\right| \in K(N) f^{N} A\right)$.

We will show that, for any $\epsilon>0$ small enough and $M>0$ large enough, we have

$$
P_{N}(i, y, f, K(N)) \sim(2 \pi)^{-1 / 2} 2 K(N) f^{N}|A| \alpha_{i+1}^{N} \cdots \alpha_{n}^{N} e^{-y^{2} N / 2}, \text { as } N \rightarrow \infty
$$

uniformly for the parameter $y$ in the domain

$$
\max _{m=i+1, \ldots, n} \frac{\left(a_{i}+\cdots+a_{n}\right)\left(2 \ln \alpha_{m}+\cdots+2 \ln \alpha_{n}+2 \ln f+\epsilon\right)}{a_{m}+\cdots+a_{n}} \leq y^{2} \leq M .
$$

Then, the probability $(2.5)$ equals $\left(1-P_{N}(i, y, f, K(N))\right)^{\alpha_{i}^{N}}$, where the asymptotics of the quantity $P_{N}(i, y, f, K(N))$ is established in (2.8). Moreover, by the assumption (2.4),

$$
P_{N}(i, y, f, K(N)) \leq(2 \pi)^{-1 / 2} 2 K(N)|A| \exp (-\epsilon N / 2) \rightarrow 0 .
$$

Then the elementary inequality, $-x-x^{2} \leq \ln (1-x) \leq-x$, that holds for $|x|<1 / 2$, leads to (2.6).

Therefore we concentrate on the proof of the asymptotics (2.8). Let $X$ be a standard Gaussian random variable. Then

$P_{N}(n, y, f, K(N))=\mathbb{P}\left(|X-y \sqrt{N}| \in K(N) f^{N} A\right) \sim(2 \pi)^{-1 / 2} 2 K(N) f^{N}|A| e^{-y^{2} N / 2}, \quad N \rightarrow \infty$

uniformly for $y^{2} \leq M$. This implies (2.8) for $i=n$. Note also that

$$
P_{N}(i, y, f, K(N)) \leq \alpha_{i+1}^{N} \cdots \alpha_{n}^{N} \mathbb{P}\left(|X-y \sqrt{N}| \in K(N) f^{N} A\right)
$$


so that the upper bound for (2.8) is immediate. We will establish the lower bound by induction downwards from $i=n$ to $i=1$, using the identity

$$
\begin{aligned}
& P_{N}(i, y, f, K(N))=\int_{-\infty}^{\infty} \frac{d t e^{-t^{2} / 2}}{\sqrt{2 \pi}}(1-[1- \\
& \left.\left.\quad-P_{N}\left(i+1, \frac{\sqrt{a_{i}+\cdots+a_{n}} y \sqrt{N}-\sqrt{a_{i}} t}{\sqrt{N\left(a_{i+1}+\cdots+a_{n}\right)}}, f, \frac{\sqrt{a_{i}+\cdots+a_{n}}}{\sqrt{a_{i+1}+\cdots+a_{n}}} K(N)\right)\right]^{\alpha_{i+1}^{N}}\right) .
\end{aligned}
$$

By the induction hypothesis for $i+1$,

$$
\begin{aligned}
& P_{N}\left(i+1, \frac{\sqrt{a_{i}+\cdots+a_{n}} y-\sqrt{a_{i}} t}{\sqrt{N\left(a_{i+1}+\cdots+a_{n}\right)}}, f, \frac{\sqrt{a_{i}+\cdots+a_{n}}}{\sqrt{a_{i+1}+\cdots+a_{n}}} K(N)\right) \\
& \quad \sim(2 \pi)^{-1 / 2} \frac{\sqrt{a_{i}+\cdots+a_{n}}}{\sqrt{a_{i+1}+\cdots+a_{n}}} 2 K(N) f^{N}|A| \alpha_{i+2}^{N} \alpha_{i+3}^{N} \cdots \alpha_{n}^{N} e^{-\frac{\left(\sqrt{a_{i}+\cdots+a_{n}} y \sqrt{N}-\sqrt{a_{i}} t\right)^{2}}{2\left(a_{i+1}+\cdots+a_{n}\right)}}
\end{aligned}
$$

uniformly for all $y, t$ that satisfying

$$
\begin{gathered}
\max _{m=i+2, \ldots, n} \frac{\left(a_{i+1}+\cdots+a_{n}\right)\left(2 \ln \alpha_{m}+\cdots+2 \ln \alpha_{n}+2 \ln f+\epsilon_{i+1}\right)}{a_{m}+\cdots+a_{n}} \\
\leq\left(\frac{\sqrt{a_{i}+\cdots+a_{n}} y \sqrt{N}-\sqrt{a_{i}} t}{\sqrt{N\left(a_{i+1}+\cdots+a_{n}\right)}}\right)^{2} \leq M_{i+1},
\end{gathered}
$$

for any $\epsilon_{i+1}>0$ small enough and $M_{i+1}>0$ large enough. The right-hand side of this inequality reads

$$
\begin{aligned}
\sqrt{N} T_{1}^{-}(y) & \equiv \sqrt{N} \frac{\sqrt{a_{i}+\cdots+a_{n}} y-\sqrt{a_{i+1}+\cdots+a_{n}} M_{i+1}}{\sqrt{a_{i}}} \leq t \\
& \leq \sqrt{N} \frac{\sqrt{a_{i}+\cdots+a_{n}} y+\sqrt{a_{i+1}+\cdots+a_{n}} M_{i+1}}{\sqrt{a_{i}}}=\sqrt{N} T_{1}^{+}(y) .
\end{aligned}
$$

Obviously, the left-hand side of (2.15) holds for all $t \in(-\infty, \infty)$, if $\ln \alpha_{n}+\cdots+\ln \alpha_{i+2}+$ $2 \ln f<0$ and $\epsilon_{i+1}$ is small enough. Otherwise, it holds, if either

$$
\begin{aligned}
t \geq & \frac{\sqrt{N}}{\sqrt{a_{i}}} \max _{\substack{m=i+2, \ldots, n: \\
\ln \alpha_{n}+\cdots+\ln \alpha_{m}+2 \ln f \geq 0}}\left(\sqrt{a_{i}+\cdots+a_{n}} y\right. \\
& \left.+\frac{a_{i+1}+\cdots+a_{n}}{\sqrt{a_{m}+\cdots+a_{n}}} \sqrt{2 \ln \alpha_{m}+\cdots+2 \ln \alpha_{n}+2 \ln f+\epsilon_{i+1}}\right) \equiv \sqrt{N} T_{2}^{+}(y),
\end{aligned}
$$

or

$$
\begin{aligned}
t \leq & \frac{\sqrt{N}}{\sqrt{a_{i}}} \min _{\substack{m=i+2, \ldots, n: \\
\ln \alpha_{n}+\cdots+\ln \alpha m+2 \ln f \geq 0}}\left(\sqrt{a_{i}+\cdots+a_{n}} y\right. \\
& \left.-\frac{a_{i+1}+\cdots+a_{n}}{\sqrt{a_{m}+\cdots+a_{n}}} \sqrt{2 \ln \alpha_{m}+\cdots+2 \ln \alpha_{n}+2 \ln f+\epsilon_{i+1}}\right) \equiv \sqrt{N} T_{2}^{-}(y) .
\end{aligned}
$$


Let us put for convenience $T_{2}^{+}(y)=-\infty$ and $T_{2}^{-}(y)=\infty$, if $2 \ln \alpha_{n}+\cdots+2 \ln \alpha_{i+2}+2 \ln f<0$. Finally,

$$
\alpha_{i+1}^{N} P_{N}\left(i+1, \frac{\sqrt{a_{i}+\cdots+a_{n}} y-\sqrt{a_{i}} t}{\sqrt{N\left(a_{i+1}+\cdots+a_{n}\right)}}, f, \frac{\sqrt{a_{i}+\cdots+a_{n}}}{\sqrt{a_{i+1}+\cdots+a_{n}}} K\right) \rightarrow 0,
$$

uniformly in the domain where

$$
\left(\frac{\sqrt{a_{i}+\cdots+a_{n}} y \sqrt{N}-\sqrt{a_{i}} t}{\sqrt{N\left(a_{i+1}+\cdots+a_{n}\right)}}\right)^{2} \geq 2 \ln \alpha_{i+1}+\cdots+2 \ln \alpha_{n}+2 \ln f+\epsilon_{i+1} .
$$

This domain is equivalent to $-\infty<t<+\infty$, if $2 \ln \alpha_{n}+\cdots+2 \ln \alpha_{i+1}+2 \ln f<0$ and $\epsilon_{i+1}>0$ is small enough. Otherwise, it is reduced to the union of the domains

$$
\begin{aligned}
t & \geq \frac{\sqrt{N}}{\sqrt{a_{i}}}\left(\sqrt{a_{i}+\cdots a_{n}} y+\sqrt{\left(a_{i+1}+\cdots+a_{n}\right)\left(2 \ln \alpha_{i+1}+\cdots+2 \ln \alpha_{n}+2 \ln f+\epsilon_{i+1}\right)}\right) \\
& \equiv T_{3}^{+}(y) \sqrt{N}
\end{aligned}
$$

and

$$
\begin{aligned}
t & \leq \frac{\sqrt{N}}{\sqrt{a_{i}}}\left(\sqrt{a_{i}+\cdots a_{n}} y-\sqrt{\left(a_{i+1}+\cdots+a_{n}\right)\left(2 \ln \alpha_{i+1}+\cdots+2 \ln \alpha_{n}+2 \ln f+\epsilon_{i+1}\right)}\right) . \\
& \equiv T_{3}^{+}(y) \sqrt{N}
\end{aligned}
$$

Then, using the elementary inequalities

$$
-x-x^{2} \leq \ln (1-x) \leq-x, \quad 1+x \leq e^{x} \leq 1+x+x^{2} \text { for }|x|<1 / 2
$$

it is easy to deduce from (2.13), (2.14), and (2.19) the following asymptotic lower bound, if $2 \ln \alpha_{n}+\cdots+2 \ln \alpha_{i+1}+2 \ln f \geq 0$ :

$$
\begin{aligned}
& P(i, y, f, K(N)) \geq(2 \pi)^{-1} \frac{\sqrt{a_{i}+\cdots+a_{n}}}{\sqrt{a_{i+1}+\cdots+a_{n}}} 2 K(N) f^{N} \alpha_{i+1}^{N} \alpha_{i+2}^{N} \alpha_{i+3}^{N} \cdots \alpha_{n}^{N} \\
& \times\left(\int_{T_{1}^{-}(y) \sqrt{N}}^{\min \left(T_{2}^{-}(y), T_{3}^{-}(y)\right) \sqrt{N}} \int_{\max \left(T_{2}^{+}(y), T_{3}^{+}(y)\right) \sqrt{N}}^{T_{1}^{+}(y) \sqrt{N}}\right) e^{-\frac{\left(\sqrt{a_{i}+\cdots+a_{n}} y \sqrt{N}-\sqrt{a_{i}} t\right)^{2}}{2\left(a_{i+1}+\cdots+a_{n}\right)}} e^{-t^{2} / 2} d t .
\end{aligned}
$$

If $2 \ln \alpha_{i+1}+\cdots+2 \ln \alpha_{n}+2 \ln f<0$, then from the same assertions we deduce the same bound, but with the domain of integration ranging over the entire interval $\left[T_{1}^{-}(y) \sqrt{N}, T_{1}^{+}(y) \sqrt{N}\right]$. By the change of variables,

$$
s=\frac{\sqrt{a_{i}+\cdots+a_{n}} t-\sqrt{a_{i}} y}{\sqrt{a_{i+1}+\cdots+a_{n}}}
$$


the right-hand side of (2.24) equals

$$
\frac{2 K(N)}{2 \pi} f^{N} \alpha_{i+1}^{N} \alpha_{i+2}^{N} \alpha_{i+3}^{N} \cdots \alpha_{n}^{N} e^{-y^{2} N / 2}\left(\int_{S_{1}^{-}(y) \sqrt{N}}^{\min \left(S_{2}^{-}(y), S_{3}^{-}(y)\right) \sqrt{N}}+\int_{\max \left(S_{2}^{+}(y), S_{3}^{+}(y)\right) \sqrt{N}}^{S_{1}^{+}(y) \sqrt{N}}\right) e^{-s^{2} / 2} d s
$$

where

$$
\begin{aligned}
S_{1}^{-}(y), S_{1}^{+}(y) & =\frac{\sqrt{a_{i+1}+\cdots+a_{n}} y \pm \sqrt{a_{i}+\cdots+a_{n}} M_{i+1}}{\sqrt{a_{i}}} \\
S_{2}^{-}(y)=\min _{\substack{m=i+1, \ldots, n: \\
\ln \alpha_{n}+\cdots+\ln \alpha_{l}+2 \ln f \geq 0}} & \sqrt{\left(a_{i+1}+\cdots+a_{n}\right) / a_{i}} \\
& \times\left(y-\frac{\sqrt{a_{i}+\cdots+a_{n}}}{\sqrt{a_{m}+\cdots+a_{n}}} \sqrt{\ln \alpha_{m}+\cdots+\ln \alpha_{n}+\ln f+\epsilon_{i+1}}\right), \\
S_{2}^{+}(y)=\max _{\substack{m=i+1, \ldots, n: \\
\ln \alpha_{n}+\cdots+\ln \alpha_{m}+2 \ln f \geq 0}} & \sqrt{\left(a_{i+1}+\cdots+a_{n}\right) / a_{i}} \\
& \times\left(y+\frac{\sqrt{a_{i}+\cdots+a_{n}}}{\sqrt{a_{m}+\cdots+a_{n}}} \sqrt{\ln \alpha_{l}+\cdots+\ln \alpha_{n}+\ln f+\epsilon_{i+1}}\right),
\end{aligned}
$$

if $T_{2}^{ \pm}(y)$ are finite, and, of course, $S_{2}^{+}(y)=-\infty$, if $T_{2}^{+}(y)=-\infty, S_{2}^{-}(y)=+\infty$, if $T_{2}^{-}(y)=$ $+\infty$, and finally

$$
S_{3}^{ \pm}(y)=\frac{\sqrt{a_{i+1}+\cdots+a_{n}} y \pm \sqrt{a_{i}+\cdots+a_{n}} \sqrt{\ln \alpha_{i+1}+\cdots+\ln \alpha_{n}+\ln f+\epsilon_{i+1}}}{\sqrt{a_{i}}}
$$

Now let us take any $\epsilon>\epsilon_{i+1}$ and $M=M_{i+1}$ Then, there exist $\delta>0$ and $Q>0$, such that, for all $y \geq 0$ satisfying $(2.9)$, we have $S_{1}^{-}(y) \leq-Q$ and $\min \left(S_{2}^{-}(y), S_{3}^{-}(y)\right) \geq \delta$; and for all $y<0$ satisfying $(2.9)$, we have $S_{1}^{+}(y) \geq Q$ and $\max \left(S_{2}^{+}(y), S_{3}^{+}(y)\right) \leq-\delta$. Hence

$$
(2 \pi)^{-1 / 2}\left(\int_{S_{1}^{-}(y) \sqrt{N}}^{\min \left(S_{2}^{-}(y), S_{3}^{-}(y)\right) \sqrt{N}}+\int_{\max \left(S_{2}^{-}(y), S_{3}^{-}(y)\right) \sqrt{N}}^{S_{1}^{+}(y) \sqrt{N}}\right) e^{-s^{2} / 2} d s \geq(2 \pi)^{-1 / 2} \int_{-Q \sqrt{N}}^{\delta \sqrt{N}} e^{-s^{2} / 2} d s \rightarrow 1,
$$

as $N \rightarrow \infty$. In the case when $2 \ln _{n}+\cdots+2 \ln \alpha_{i+1}+2 \ln f<0$, we have the analogue of $(2.24)$ with the integral over $\left[T_{1}^{-}(y) \sqrt{N}, T_{1}^{+}(y) \sqrt{N}\right]$, and by the same change we get the bound

$$
(2 \pi)^{-1 / 2} \int_{S_{1}^{-}(y) \sqrt{N}}^{S_{1}^{+}(y) \sqrt{N}} e^{-s^{2} / 2} d s \geq(2 \pi)^{-1 / 2} \int_{-Q \sqrt{N}}^{Q \sqrt{N}} e^{-s^{2} / 2} d s \rightarrow 1, \quad N \rightarrow \infty .
$$


Since $\epsilon_{i+1}$ [resp. $M_{i+1}$ ] could be chosen arbitrarily small [resp. large], by the induction hypothesis, the estimates (2.24), (2.26), and (2.31), (2.32) show that, for any $\epsilon>0$ small enough, and $M>0$ large enough, the assertion (2.8) holds uniformly in the domain (2.9). This finishes the proof of the lemma. $\diamond$

Lemma 2.1 implies the next lemma.

Lemma 2.2: Let $l \in\{0, \ldots, k-1\}$, c be with $|c|<\sqrt{2 \ln \bar{\alpha}_{l+1}\left(\bar{a}_{l+1}+\cdots+\bar{a}_{k}\right) / \bar{a}_{l+1}}$. For any $\epsilon, \delta>0$ small enough, and $M>0$, there exists $N_{0}=N_{0}(\epsilon, \delta, M)$, such that, for all $N \geq N_{0}$, the probability

$$
\mathbb{P}\left(\forall \check{\sigma} \in\{-1,1\}^{\left(1-w_{l}\right) N}:\left|\frac{\check{X}_{\tilde{\sigma}}^{J_{l}}}{\sqrt{\bar{a}_{l+1}+\cdots+\bar{a}_{k}}}-(|c|+z) \sqrt{N}\right| \notin K(N) e^{c^{2} N / 2}\left(\bar{\alpha}_{l+1} \cdots \bar{\alpha}_{k}\right)^{-N} A\right)
$$

is bounded from above and below, respectively, by

$$
\exp \left(-(1 \pm \delta)(2 \pi)^{-1 / 2} 2 K(N)|A| e^{-\left(2|c| z+z^{2}\right) N / 2}\right)
$$

for any $-\epsilon<z<M$.

Proof. If $|c|<\sqrt{2 \ln \bar{\alpha}_{l+1}\left(\bar{a}_{l+1}+\cdots+\bar{a}_{k}\right) / \bar{a}_{l+1}}$, then by (1.5) we have $e^{c^{2} / 2}\left(\bar{\alpha}_{l+1} \cdots \bar{\alpha}_{k}\right)^{-1}<$ 1 and with some $\epsilon_{0}>0$ small enough:

$$
\begin{gathered}
\max \left(\max _{m=J_{l}+2, \ldots, n} \frac{\left(a_{J_{l}+1}+\cdots+a_{n}\right)\left(2 \ln \alpha_{m}+\cdots+2 \ln \alpha_{n}+2\left(c^{2} / 2-\ln \left(\bar{\alpha}_{J_{l+1}} \cdots \bar{\alpha}_{J_{k}}\right)\right)+\epsilon_{0}\right)}{a_{m}+\cdots+a_{n}},\right. \\
\left(2 \ln \alpha_{J_{l}+2}+\cdots+2 \ln \alpha_{n}+2\left(c^{2} / 2-\ln \left(\bar{\alpha}_{J_{l+1}} \cdots \bar{\alpha}_{J_{k}}\right)\right)+\epsilon_{0}\right)<c^{2}
\end{gathered}
$$

This last inequality remains true with $c^{2}$ replaced in the left-hand side by $(|c|+z)^{2}$ if $z>-\epsilon$ with $\epsilon>0$ small enough. Then Lemma 2.1 applies with $i=J_{l}+1$ and $f=e^{c^{2} / 2}\left(\bar{\alpha}_{l+1} \cdots \bar{\alpha}_{k}\right)^{-1}$ and gives the asymptotics $(2.34) \cdot \diamond$

Lemma 2.2 with $l=0, z=0, K(N)=\sqrt{2 \pi} / 2$ implies immediately the convergence of the avoidance function (2.1) in the case $l=0$. To conclude the proof of (1.12), let us note that

$$
\mathbb{E} \mathcal{M}_{N}^{0}(A)=\sum_{\sigma \in \Sigma_{N}} \mathbb{P}\left(\left|X_{\sigma}-c_{N} \sqrt{N}\right| \in 2^{-N-1}(2 \pi) e^{c_{N}^{2} N / 2} A\right)
$$

is the sum of $2^{N}$ identical terms, each of them being $2^{-N}|A|(1+o(1))$ by the trivial estimate for standard Gaussian random variables (2.11). Then (2.36) converges to $|A|$ and the proof of (1.12) is finished. 
To prove the convergence of the avoidance function (2.1) in the case $l \geq 1$, let us write the event $\left\{\mathcal{M}_{N}^{l}(A)=0\right\}$ in terms of the functions $U_{l, N}$ defined in (1.21) as

$$
\begin{aligned}
& \left\{\mathcal{M}_{N}^{l}(A)=0\right\} \\
& =\left\{\forall \hat{\sigma} \in \Sigma_{w_{l} N}, \check{\sigma} \in \Sigma_{\left(1-w_{l}\right) N}:\left|\check{X}_{\hat{\sigma} \check{\sigma}}^{J_{l}}-\sqrt{N}\left[\tilde{c}_{l}+N^{-1}\left(\Gamma_{l, N}-U_{l, N}^{-1}\left(\widehat{X}_{\hat{\sigma}}^{J_{l}}\right)\right)\right]\right| \notin R_{l}(N)^{-1} A\right\}
\end{aligned}
$$

with the abbreviations (1.20), (1.22), (1.26). Let us introduce the following event with a parameter $y>0$ :

$$
\begin{aligned}
B_{N}^{l}(y)=\{ & \forall j=1, \ldots, l, \forall \hat{\sigma} \in \Sigma_{w_{l} N}: \\
& \left.2 \Gamma_{j, N}-2 N d_{j}-\left(\gamma_{1}+\cdots+\gamma_{j}\right) y<U_{j, N}^{-1}\left(\widehat{X}_{\hat{\sigma}}^{J_{j}}\right)<y\left(\gamma_{1}+\cdots+\gamma_{j}\right)\right\}
\end{aligned}
$$

By the convergence (1.23) to (1.24), the property (1.6) and the symmetry of the standard Gaussian distribution, the probability of the complementary event satisfies the following bound:

$$
\lim \sup _{N \rightarrow \infty} \mathbb{P}\left(\bar{B}_{N}^{l}(y)\right) \leq 2 \exp (-h y)
$$

with some constant $h>0$. Now, let us fix any arbitrarily large $y>0$ and consider

$$
\begin{aligned}
\mathbb{P}\left(\mathcal{M}_{N}^{l}(A)=0\right)= & \mathbb{E}\left[\mathbb{I}_{\left\{B_{N}^{l}(y)\right\}} \mathbb{E}\left(\mathbb{I}_{\left\{\mathcal{M}_{N}^{l}(A)=0\right\}} \mid \widehat{X}_{\hat{\sigma}}^{J_{j}}, \forall_{j=1}^{l}, \forall \widehat{\sigma} \in \Sigma_{w_{l} N}\right)\right] \\
& +\mathbb{E}\left[\mathbb{I}_{\left\{\bar{B}_{N}^{l}(y)\right\}} \mathbb{E}\left(\mathbb{I}_{\left\{\mathcal{M}_{N}^{l}(A)=0\right\}} \mid \widehat{X}_{\hat{\sigma}}^{J_{j}}, \forall_{j=1}^{l}, \forall \widehat{\sigma} \in \Sigma_{w_{l} N}\right)\right]
\end{aligned}
$$

Due to the representation (2.37), the conditional expectation $\mathbb{E}\left(\mathbb{I}_{\left\{\mathcal{M}_{N}^{l}(A)=0\right\}} \mid \widehat{X}_{\hat{\sigma}}^{J_{j}}, \forall_{j=1}^{l}, \forall \widehat{\sigma} \in\right.$ $\left.\Sigma_{w_{l} N}\right)$ can be viewed as the product over $\widehat{\sigma} \in \Sigma_{w_{l} N}$ of the quantities (2.33) with

$$
|c|=\frac{\tilde{c}_{l}}{\sqrt{\bar{a}_{l+1}+\cdots+\bar{a}_{k}}}, \quad K(N)=\frac{\sqrt{2 \pi}}{2} \prod_{j=1}^{l}\left(4 N \pi \ln \bar{\alpha}_{j}\right)^{\beta_{l} \gamma_{j} / 2}
$$

and

$$
z=z(\hat{\sigma})=\left(\bar{a}_{l+1}+\cdots+\bar{a}_{k}\right)^{-1 / 2} N^{-1}\left(\Gamma_{l, N}-U_{l, N}^{-1}\left(\widehat{X}_{\hat{\sigma}}^{J_{l}}\right)\right), \quad \hat{\sigma} \in \Sigma_{w_{l} N}
$$

Furthermore, on $B_{N}^{l}(y)$, we have $z(\widehat{\sigma}) \in\left(-\epsilon, \frac{2 d_{l}}{\sqrt{\bar{a}_{l+1}+\cdots+\bar{a}_{k}}}+\epsilon\right) \forall \hat{\sigma} \in \Sigma_{w_{l} N}$ (with some small enough $\epsilon>0)$, so that Lemma 2.2 applies to $\mathbb{I}_{\left\{B_{N}^{l}(y)\right\}} \mathbb{E}\left(\mathbb{I}_{\left\{\mathcal{M}_{N}^{l}(A)=0\right\}} \mid \widehat{X}_{\hat{\sigma}}^{J_{j}}, \forall_{j=1}^{l}, \forall \widehat{\sigma} \in \Sigma_{w_{l} N}\right)$. Hence, by (2.40) and by Lemma 2.2, for any $\delta>0$ small enough, there exists $N_{0}(\delta, y)$ such 
that for all $N \geq N_{0}$

$$
\begin{aligned}
& \mathbb{E}\left[\prod_{\hat{\sigma} \in \Sigma_{w_{l} N}} \exp \left(-(1-\delta)(2 \pi)^{-1 / 2} 2 K(N)|A| e^{-\left(2|c| z(\hat{\sigma})+z^{2}(\hat{\sigma})\right) N / 2}\right)\right]+\mathbb{P}\left(\bar{B}_{N}^{l}(y)\right) \\
& \geq \mathbb{E}\left[\mathbb{I}_{\left\{B_{N}^{l}(y)\right\}} \prod_{\hat{\sigma} \in \Sigma_{w_{l} N}} \exp \left(-(1-\delta)(2 \pi)^{-1 / 2} 2 K(N)|A| e^{-\left(2|c| z(\hat{\sigma})+z^{2}(\hat{\sigma})\right) N / 2}\right)\right]+\mathbb{P}\left(\bar{B}_{N}^{l}(y)\right) \\
& \geq \mathbb{P}\left(\mathcal{M}_{N}^{l}(A)=0\right) \\
& \geq \mathbb{E}\left[\mathbb{I}_{\left\{B_{N}^{l}(y)\right\}} \prod_{\hat{\sigma} \in \Sigma_{w_{l} N}} \exp \left(-(1+\delta)(2 \pi)^{-1 / 2} 2 K(N)|A| e^{-\left(2|c| z(\hat{\sigma})+z^{2}(\hat{\sigma})\right) N / 2}\right)\right] \\
& \geq \mathbb{E}\left[\prod_{\hat{\sigma} \in \Sigma_{w_{l} N}} \exp \left(-(1+\delta)(2 \pi)^{-1 / 2} 2 K(N)|A| e^{-\left(2|c| z(\hat{\sigma})+z^{2}(\hat{\sigma})\right) N / 2}\right)\right]-\mathbb{P}\left(\bar{B}_{N}^{l}(y)\right) .
\end{aligned}
$$

Using the convergence (1.23) to (1.24), we derive that for any $y>0$ large enough and $\delta>0$ small enough

$$
\begin{aligned}
& \mathbb{E} \prod_{\left(x_{1}, \ldots, x_{l}\right) \in \mathcal{P}_{l}} \exp \left(-(1-\delta)|A| e^{\beta_{l}\left(\gamma_{1} x_{1}+\cdots+\gamma_{l} x_{l}\right)}\right)+\lim \sup _{N \rightarrow \infty} \mathbb{P}\left(\bar{B}_{N}^{l}(y)\right) \\
& \geq \lim \sup _{N \rightarrow \infty} \mathbb{P}\left(\mathcal{M}_{N}(A)=0\right) \geq \lim \inf _{N \rightarrow \infty} \mathbb{P}\left(\mathcal{M}_{N}(A)=0\right) \\
& \geq \mathbb{E} \prod_{\left(x_{1}, \ldots, x_{l}\right) \in \mathcal{P}_{l}} \exp \left(-(1+\delta)|A| e^{\beta_{l}\left(\gamma_{1} x_{1}+\cdots+\gamma_{l} x_{l}\right)}\right)-\lim \sup _{N \rightarrow \infty} \mathbb{P}\left(\bar{B}_{N}^{l}(y)\right) .
\end{aligned}
$$

Thus (2.44) and (2.39) imply the following bounds:

$$
\begin{aligned}
& \mathbb{E} \exp \left(-(1-\delta)|A| \Lambda_{l}\right)+2 \exp (-h y) \geq \lim \sup _{N \rightarrow \infty} \mathbb{P}\left(\mathcal{M}_{N}^{l}(A)=0\right) \\
& \left.\geq \lim \inf _{N \rightarrow \infty} \mathbb{P}\left(\mathcal{M}_{N}^{l}(A)=0\right) \geq \mathbb{E} \exp \left(-(1+\delta)|A| \Lambda_{l}\right)\right)-2 \exp (-h y) .
\end{aligned}
$$

Since $y>0$ can be chosen arbitrarily large and $\delta>0$ fixed arbitrarily small, this finishes the proof of the convergence of the avoidance function (2.1) in the case of $l=1,2, \ldots, k-1$.

To proceed with the proof of tightness (2.3), we need the following lemma.

Lemma 2.3: Let $l \in\{0, \ldots, k-1\},|c|<\sqrt{2 \ln \bar{\alpha}_{l+1}\left(\bar{a}_{l+1}+\cdots+\bar{a}_{k}\right) / \bar{a}_{l+1}}, K(N)>0$ is polynomial in $N, z \in \mathbb{R}$. For any segment $B \subset \mathbb{R}_{+}$, let us define an integer-valued random variable

$$
\begin{aligned}
& T_{l, N}^{c, z}, K(N)(B) \\
& =\#\left\{\check{\sigma} \in \Sigma_{\left(1-w_{l}\right) N}:\left|\frac{\check{X}_{\check{\sigma}}^{J_{l}}}{\sqrt{\bar{a}_{l+1}+\cdots+\bar{a}_{k}}}-\sqrt{N}(|c|+z)\right| \in K(N) e^{c^{2} N / 2}\left(\bar{\alpha}_{l+1} \cdots \bar{\alpha}_{k}\right)^{-N} B\right\} .
\end{aligned}
$$


(i) For any bounded segment $A \subset \mathbb{R}_{+}$, any $\epsilon, \delta>0$ small enough and $M>0$ there exists $N_{0}=N_{0}(\delta, M, \epsilon)$ such that for all $N \geq N_{0}$, for any $B \subset A$ and any $\left.z \in\right]-\epsilon, M[$ we have:

$$
\mathbb{P}\left(T_{l, N}^{c, z, K(N)}(B) \geq 1\right) \leq(1+\delta)|B| K(N)(2 / \sqrt{2 \pi}) e^{-\left(2|c| z+z^{2}\right) N / 2} .
$$

(ii) For any bounded segment $A \subset \mathbb{R}_{+}$, any $\delta>0$ small enough, $K>0$ large enough and $M>0$ there exists $N_{0}=N_{0}(\delta, M, K)$ such that for all $N \geq N_{0}$, for any segment $B \subset A$ with $|B|<K^{-1}$ and for any

$$
\left.z=z_{N} \in\right] \frac{\ln (2 K(N) / \sqrt{2 \pi})-\ln K}{|c| N}, M[
$$

we have:

$$
\begin{aligned}
& \mathbb{P}\left(T_{l, N}^{c, z, K(N)}(B) \geq 2\right) \\
& \leq \delta|B| K(N)(2 / \sqrt{2 \pi}) e^{-\left(2|c| z+z^{2}\right) N / 2}+\left(|B| K(N)(2 / \sqrt{2 \pi}) e^{-\left(2|c| z+z^{2}\right) N / 2}\right)^{2} / 2 .
\end{aligned}
$$

Remark: The bound (2.49) is far from being the optimal one, but it is enough for our purpose. Therefore, we do not prove a precise bound that requires much more tedious computations.

Proof. The right-hand side of (2.47) is bounded from above by

$$
\left(\bar{\alpha}_{l+1} \cdots \bar{\alpha}_{k}\right)^{N} \mathbb{P}\left(|X-\sqrt{N}(|c|+z)| \in K(N) e^{c^{2} N / 2}\left(\bar{\alpha}_{l+1} \cdots \bar{\alpha}_{k}\right)^{-N} B\right)
$$

with $X$ a standard Gaussian random variable. Since by the assumption of the lemma and by (1.5) we have $e^{c^{2} / 2}\left(\bar{\alpha}_{l+1} \cdots \bar{\alpha}_{k}\right)^{-1}<1$, then (2.47) is obvious from the trivial estimate (2.11).

To prove (ii), note that $\mathbb{E} T_{l, N}^{c, z, K(N)}(B)$ just equals (2.50), whence

$$
\mathbb{E} T_{l, N}^{c, z, K(N)}(B) \leq(1+\delta)|B| K(N)(2 / \sqrt{2 \pi}) e^{-\left(2|c| z+z^{2}\right) N / 2}
$$

Finally

$$
\mathbb{P}\left(T_{l, N}^{c, z, K(N)}(B) \geq 2\right) \leq \mathbb{E} T_{l, N}^{c, z, K(N)}(B)-\left(1-\mathbb{P}\left(T_{l, N}^{c, z, K(N)}(B)=0\right)\right)
$$

where by Lemma $2.2 \mathbb{P}\left(T_{l, N}^{c, z, K(N)}(B)=0\right)$ is bounded from above by the exponent (2.34). The assumption (2.48) and the fact that $|B|<1 / K$ assure that the argument of this exponent is smaller than 1 by absolute value, i.e.

$$
0<(1-\delta)|B| K(N)(2 / \sqrt{2 \pi}) e^{-\left(2|c| z+z^{2}\right) N / 2}<1-\delta .
$$


Then (2.52), (2.51), the bound (2.34) with (2.53) and the elementary fact that $e^{-x} \leq 1-x+$ $x^{2} / 2$ for $0<x<1$ yield the estimate $(2.49)$. $\diamond$

We are now ready to prove the tightness (2.3) of the family $\left\{\mathcal{M}_{N}^{l}\right\}_{N=1}^{\infty}$ for $l=1, \ldots, k-1$. For a given $\epsilon>0$, let us first fix $y$ large enough and $N_{1}(y)$ such that

$$
\mathbb{P}\left(\bar{B}_{N}^{l}(y)\right)<\epsilon / 4 \quad \forall N \geq N_{1}=N_{1}(y)
$$

which is possible due to (2.39). Now let us split the segment $A=[a, b]$ into $R$ disjoint segments $A_{1}, \ldots, A_{R}$ of size $(b-a) / R, R>1$. Then

$$
\begin{aligned}
& \mathbb{P}\left(\left\{\mathcal{M}_{N}^{l}(A)>R\right\} \cap B_{N}^{l}(y)\right) \leq \sum_{i=1}^{R} \mathbb{P}\left(\left\{\mathcal{M}_{N}^{l}\left(A_{i}\right) \geq 2\right\} \cap B_{N}^{l}(y)\right) \\
& \leq \sum_{i=1}^{R} \sum_{\hat{\sigma} \in \Sigma_{w_{l}} N} \mathbb{P}\left(C_{N}^{l}\left(A_{i}, \widehat{\sigma}\right) \cap B_{N}^{l}(y, \widehat{\sigma})\right) \\
& \quad+\sum_{i=1}^{R} \sum_{\hat{\tau}, \hat{\eta} \in \Sigma_{w_{l} N}, \hat{\tau} \neq \hat{\eta}} \mathbb{P}\left(D_{N}^{l}\left(A_{i}, \widehat{\tau}\right) \cap D_{N}^{l}\left(A_{i}, \widehat{\eta}\right) \cap B_{N}^{l}(y, \widehat{\tau}) \cap B_{N}^{l}(y, \widehat{\eta})\right)
\end{aligned}
$$

where

$$
\begin{gathered}
C_{N}^{l}\left(A_{i}, \widehat{\sigma}\right)=\left\{\exists \check{\eta}, \check{\tau} \in \Sigma_{\left(1-w_{l}\right) N}, \check{\eta} \neq \check{\tau}:\right. \\
\left.\left|\check{X}_{\hat{\sigma} \check{\sigma}}^{J_{l}}-\sqrt{N}\left[\widetilde{c}_{l}+N^{-1}\left(\Gamma_{l, N}-U_{l, N}^{-1}\left(\widehat{X}_{\hat{\sigma}}^{J_{l}}\right)\right)\right]\right| \in R_{l}(N)^{-1} A_{i} \text { for } \check{\sigma}=\check{\eta}, \check{\sigma}=\check{\tau}\right\}, \\
D_{N}^{l}\left(A_{i}, \widehat{\sigma}\right)=\left\{\exists \check{\sigma} \in \Sigma_{\left(1-w_{l}\right) N}:\left|\check{X}_{\tilde{\sigma} \check{\sigma}}^{J_{l}}-\sqrt{N}\left[\widetilde{c}_{l}+N^{-1}\left(\Gamma_{l, N}-U_{l, N}^{-1}\left(\widehat{X}_{\tilde{\sigma}}^{J_{l}}\right)\right)\right]\right| \in R_{l}(N)^{-1} A_{i}\right\},
\end{gathered}
$$

and

$$
B_{N}^{l}(y, \widehat{\sigma})=\left\{\forall j=1, \ldots, l: 2 \Gamma_{j, N}-2 N d_{j}-\left(\gamma_{1}+\cdots+\gamma_{j}\right) y<U_{j, N}^{-1}\left(\widehat{X}_{\hat{\sigma}}^{J_{j}}\right)<y\left(\gamma_{1}+\cdots+\gamma_{j}\right)\right\}
$$

Each term in the first sum of (2.55) equals

$$
\begin{aligned}
& \mathbb{E}\left[\mathbb{I}_{\left\{B_{N}^{l}(y, \hat{\sigma})\right\}} \mathbb{E}\left(\mathbb{I}_{\left\{C_{N}^{l}\left(A_{i}, \hat{\sigma}\right)\right\}} \mid \widehat{X}_{\hat{\sigma}}^{J_{j}}, \forall_{j=1}^{l}\right)\right] \\
& =\mathbb{E}\left[\mathbb{I}_{\left\{B_{N}^{l}(y, \hat{\sigma})\right\}} \mathbb{E}\left(\mathbb{I}_{\left\{T_{l, N}^{c, z(\hat{\sigma}), K(N)}\left(A_{i}\right) \geq 2\right\}} \mid \widehat{X}_{\hat{\sigma}}^{J_{j}}, \forall_{j=1}^{l}\right)\right]
\end{aligned}
$$

with the random variables $T_{l, N}^{c, z, K(N)}$ defined in Lemma 2.3 and with parameters $c, K(N), z(\widehat{\sigma})$ defined by $(2.41)$ and $(2.42)$. Furthermore, on $B_{N}^{l}(y, \widehat{\sigma})$, the parameter $z(\widehat{\sigma})$ satisfies the condition (2.48) with the constant $K=e^{\beta_{l}\left(\gamma_{1}+\cdots+\gamma_{l}\right) y}$ and $M=2 d_{l}\left(\bar{a}_{l+1}+\cdots+\bar{a}_{k}\right)^{-1 / 2}+\epsilon$ 
with some small $\epsilon>0$. Therefore, if $\left|A_{i}\right|=(a-b) / R<e^{-\beta_{l}\left(\gamma_{1}+\cdots+\gamma_{l}\right) y}$, then the assertion (ii) of Lemma 2.3 applies to the conditional expectation in (2.58). Next, each term of the second sum of (2.55) equals

$$
\begin{aligned}
& \mathbb{E}\left[\mathbb{I}_{\left\{B_{N}^{l}(y, \hat{\eta}), B_{N}^{l}(y, \hat{\tau})\right\}} \mathbb{E}\left(\mathbb{I}_{\left\{D_{N}^{l}\left(A_{i}, \hat{\eta}\right)\right\}} \mid \widehat{X}_{\hat{\eta}}^{J_{j}}, \forall_{j=1}^{l}\right)\right. \\
& =\mathbb{E}\left[\mathbb{I}_{\left\{B_{N}^{l}(y, \hat{\eta}), B_{N}^{l}(y, \hat{\tau})\right\}} \mathbb{E}\left(\mathbb{I}_{\left\{D_{N}^{l}\left(A_{i}, \hat{\tau}\right)\right\}} \mid \widehat{X}_{\hat{\tau}}^{J_{j}, z(\hat{\eta}), K(N)}, \forall_{j=1}^{l}\right)\right] \\
& \left.\left.\quad \times \mathbb{E}_{\left.\left\{A_{i}\right) \geq 1\right\}}\left|\mathbb{X}_{\left.\hat{\eta} T_{l, N}^{c, z(\hat{\tau}), K(N)}\left(A_{i}\right) \geq 1\right\}}\right| \widehat{X}_{\hat{\tau}}^{J_{j}}, \forall_{j=1}^{l}\right)\right]
\end{aligned}
$$

where on $B_{N}^{l}(y, \hat{\eta}) \cap B_{N}^{l}(y, \hat{\tau})$ we have $-\epsilon<z(\widehat{\tau}), z(\widehat{\eta})<2 d_{l}\left(\bar{a}_{l+1}+\cdots+\bar{a}_{k}\right)^{-1 / 2}+\epsilon$ with some small $\epsilon>0$. Then the assertion (i) of Lemma 2.3 applies to the conditional expectations in (2.59). Thus by Lemma 2.3, for any $\delta>0$, there exists $N_{2}(y, \delta)$ such that for all $N \geq N_{2}$

$$
\begin{aligned}
& \sum_{i=1}^{R} \mathbb{P}\left(\left\{\mathcal{M}_{N}^{0}\left(A_{i}\right) \geq 2\right\} \cap B_{N}^{l}(y)\right) \\
& \leq \sum_{i=1}^{R} \delta(2 / \sqrt{2 \pi}) K(N)(b-a) R^{-1} \mathbb{E}\left(\sum_{\hat{\sigma} \in \Sigma_{w_{l}} N} \mathbb{I}_{\left\{B_{N}^{l}(y, \hat{\sigma})\right\}} e^{-\left(2|c| z(\hat{\sigma})+z^{2}(\hat{\sigma})\right) N / 2}\right) \\
& \quad+\sum_{i=1}^{R}(4 / 2 \pi) K(N)^{2}(b-a)^{2} R^{-2} \\
& \quad \times \mathbb{E}\left(\frac{1}{2} \sum_{\hat{\sigma} \in \Sigma_{w_{l} N}} \mathbb{I}_{\left\{B_{N}^{l}(y, \hat{\sigma})\right\}} e^{-\left(2|c| z(\hat{\sigma})+z^{2}(\hat{\sigma})\right) N}\right. \\
& \left.\quad+\sum_{\hat{\tau}, \hat{\eta} \in \Sigma_{w_{l} N}: \hat{\tau} \neq \hat{\eta}} \mathbb{I}_{\left\{B_{N}^{l}(y, \hat{\tau}), B_{N}^{l}(y, \hat{\eta})\right\}} e^{-\left(2|c| z(\hat{\tau})+z^{2}(\hat{\tau})+2|c| z(\hat{\eta})+z^{2}(\hat{\eta})\right) N / 2}\right) \\
& =\delta(b-a) I_{N}(y)+R^{-1}(b-a)^{2} J_{N}(y) / 2
\end{aligned}
$$

where

$$
\begin{gathered}
I_{N}(y)=(2 / \sqrt{2 \pi}) K(N) \mathbb{E}\left(\sum_{\hat{\sigma} \in \Sigma_{w_{l} N}} \mathbb{I}_{\left\{B_{N}^{l}(y, \hat{\sigma})\right\}} e^{-\left(2|c| z(\hat{\sigma})+z^{2}(\hat{\sigma})\right) N / 2}\right), \\
J_{N}(y)=(4 /(2 \pi)) K(N)^{2} \mathbb{E}\left(\sum_{\hat{\sigma} \in \Sigma_{w_{l} N}} \mathbb{I}_{\left\{B_{N}^{l}(y, \hat{\sigma})\right\}} e^{-\left(2|c| z(\hat{\sigma})+z^{2}(\hat{\sigma})\right) N / 2}\right)^{2} .
\end{gathered}
$$

Here, the quantity $I_{N}(y)$ converges to

$$
\begin{aligned}
& I(y)=\mathbb{E} \int_{\substack{\forall 1 \leq j \leq l: \\
\gamma_{1} x_{1}+\cdots+\gamma_{j} x_{j}<\left(\gamma_{1}+\cdots \gamma_{j}\right) y}} e^{\beta\left(\gamma_{1} x_{1}+\cdots \gamma_{l} x_{l}\right)} \mathcal{P}_{l}\left(d x_{1} \ldots, d x_{l}\right) \\
& =\int_{\substack{\forall 1 \leq j \leq l: \\
\gamma_{1} x_{1}+\cdots+\gamma_{j} x_{j}<\left(\gamma_{1}+\cdots \gamma_{j}\right) y}} e^{\beta\left(\gamma_{1} x_{1}+\cdots \gamma_{l} x_{l}\right)-x_{1}-\cdots-x_{l}} d x_{1}, \ldots, d x_{l}<\infty .
\end{aligned}
$$


Therefore, one can fix $N_{3}=N_{3}(y)$ large enough and then $\delta=\delta(y)$ so small that $\delta(b-$ a) $J_{N}^{1}(y)<\epsilon / 4, \forall N \geq N_{3}(y)$. The term $J_{N}(y)$ converges to

$$
J(y)=\mathbb{E}\left(\int_{\substack{\forall j=1, \ldots, l: \\\left(\gamma_{1} x_{1}+\cdots+\gamma_{j} x_{j}\right)<\left(\gamma_{1}+\cdots+\gamma_{j}\right) y}} e^{\beta_{l}\left(\gamma_{1} x_{1}+\cdots+\gamma_{l} x_{l}\right)} \mathcal{P}^{l}\left(d x_{1} \cdots d x_{l}\right)\right)^{2}
$$

which is finite. In fact, $J(y)$ is the sum of $l+1$ terms, the $k$ th of them being

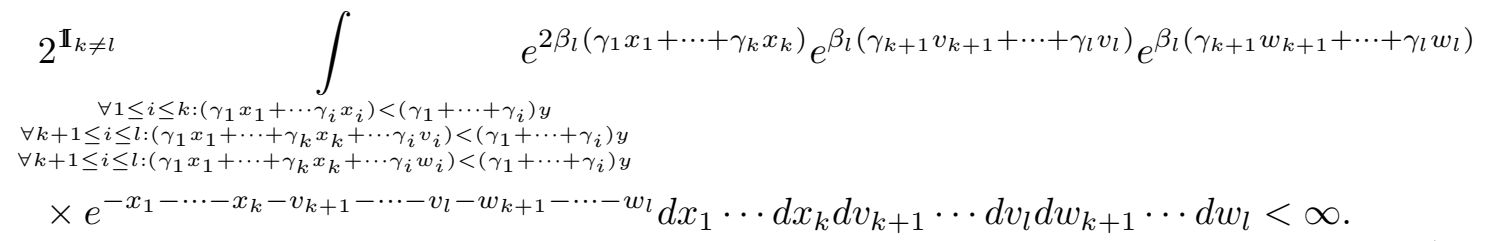

Then for any $\epsilon>0$, one can choose $N_{4}=N_{4}(y)$ such that for all $N \geq N_{4}(y)\left|J_{N}(y)-J(y)\right|<$ $\epsilon / 4$. Next, let us choose $R_{0}=R_{0}(y)>K=e^{\beta\left(\gamma_{1}+\cdots \gamma_{l}\right) y}(b-a)$ such that $(b-a)^{2} R_{0}^{-1}<1$ and also such that $(b-a)^{2} R_{0}^{-1} J(y)<\epsilon / 4$. Thus $(b-a)^{2} R^{-1} J_{N}(y) / 2<\epsilon / 2 \forall N \geq N_{4}(y)$ and $\forall R \geq R_{0}$. Hence,

$$
\sum_{i=1}^{R} \mathbb{P}\left(\left\{\mathcal{M}_{N}^{0}\left(A_{i}\right) \geq 2\right\} \cap B_{N}^{l}(y)\right)<3 \epsilon / 4 \quad \forall R \geq R_{0}, \text { and } \forall N \geq N_{2}(\delta(y), y), N_{3}(y), N_{4}(y) .
$$

Taking into account (2.54), we obtain that

$$
\mathbb{P}\left(\mathcal{M}_{N}^{l}(A)>R\right)<\epsilon \quad \forall R \geq R_{0} \text { and } \forall N \geq \max \left(N_{1}, N_{2}, N_{3}, N_{4}\right),
$$

whence

$$
\mathbb{P}\left(\mathcal{M}_{N}^{l}(A)>\max \left(R_{0}, 2^{N_{1}}, 2^{N_{2}}, 2^{N_{3}}, 2^{N_{4}}\right)\right)<\epsilon \quad \forall N \geq 1
$$

then $\mathcal{M}_{N}^{l}$ is tight.

It remains to show that the limit $\tilde{\mathcal{M}}^{l}$ of any weakly convergent subsequence of $\left\{\mathcal{M}_{N}^{l}\right\}$ is a simple process, that is very easy. Consider any segment $A=[a, b)$ and its dissecting system $\left\{A_{r, i}, i=1,2, \ldots, 2^{r}, r=1,2, \ldots\right\}$ such that $A_{1,1}=[a,(a+b) / 2)$ and $A_{1,2}=[(a+b) / 2, b)$ are obtained by splitting $[a, b)$ in the middle and the system of disjoint intervals $\left\{A_{r, i}, i=\right.$ $\left.1,2, \ldots, 2^{r}\right\}$ is obtained from $\left\{A_{r-1, i}, i=1,2, \ldots, 2^{r-1}\right\}$ by splitting similarly each segment of the latter system into two parts in the middle. It follows from the estimates (2.54) and (2.62) that for any $\epsilon>0$ there exists $N_{0}$ and $r_{0}$ such that

$$
\mathbb{P}\left(\exists i=1, \ldots, 2^{r}: \mathcal{M}_{N}^{l}\left(A_{r, i}\right) \geq 2\right)<\epsilon \quad \forall N \geq N_{0}, \forall r \geq r_{0}
$$


Then for any $\epsilon>0$ there exists $r_{0}$ such that

$$
\mathbb{P}\left(\exists i=1, \ldots, 2^{r}: \tilde{\mathcal{M}}^{l}\left(A_{r, i}\right) \geq 2\right)<\epsilon \quad \forall r \geq r_{0}
$$

Then $\tilde{\mathcal{M}}^{l}$ can have double points within $A$ with probability smaller than $\epsilon$. Since $\epsilon>0$ is arbitrary, it follows that $\tilde{\mathcal{M}}^{l}$ is simple. Thus the proof of the theorem is complete. $\diamond$

\section{REFERENCES}

[BaMe] H. Bauke and St. Mertens. Universality in the level statistics of disordered systems. Phys. Rev. E, 70:025102(R), 2004.

[BK1] A. Bovier and I. Kurkova. Derrida's generalized random energy models. I. Models with finitely many hierarchies. Ann. Inst. H. Poincaré Probab. Statist., 40(4):439-480, 2004.

[BK2] A. Bovier and I. Kurkova. Local energy statistics in disordered system: a proof of the local REM conjecture. Preprint of the University Paris 6, April (2005).

[BKL] A. Bovier, I. Kurkova, M. Lowe. Fluctuations of the free energy in the REM and the $p$-spin SK models, Ann. Probab. 30 (2002) 605-651.

[DV] D.J. Daley, D. Vere-Jones, An introduction to the theory of point processes. Springer Series in Statistics, Springer-Verlag (1988).

[Der1] B. Derrida. Random-energy model: an exactly solvable model of disordered systems. Phys. Rev. B (3), 24(5):2613-2626, 1981.

[Der2] B. Derrida. A generalization of the random energy model that includes correlations between the energies. J. Phys. Lett., 46:401-407, 1985.

[LLR] M.R. Leadbetter, G. Lindgren, and H. Rootzén. Extremes and related properties of random sequences and processes. Springer Series in Statistics. Springer-Verlag, New York, 1983.

[Ka] O. Kallenberg, Random Measures, fourth ed., Akademie Verlag, Berlin, 1986. 